\title{
Anisotropic Dark Energy and the Generalized Second Law of Thermodynamics
}

\author{
M. Sharif *and Farida Khanum ${ }^{\dagger}$ \\ Department of Mathematics, University of the Punjab, \\ Quaid-e-Azam Campus, Lahore-54590, Pakistan.
}

\begin{abstract}
We consider a Bianchi type $I$ model in which anisotropic dark energy is interacting with dark matter and anisotropic radiation. With this scenario, we investigate the validity of the generalized second law of thermodynamics. It is concluded that the validity of this law depends on different parameters like shear, skewness and equation of state.
\end{abstract}

Keywords: Bianchi Type I Model; Generalized Second Law of Thermodynamics.

PACS: $95.36 .+\mathrm{x}, 98.80 .-\mathrm{k}$

\section{Introduction}

The observational evidence that our universe is making a transition from a decelerating phase to an accelerating is the major development in cosmology. Supernova Ia data [1, 2] gave the first indication of the accelerated expansion of the universe. This was confirmed by the observations of anisotropies in the cosmic microwave background (CMB) radiations as seen in the data from

*msharif.math@pu.edu.pk

†faridakhanum.math@yahoo.com 
satellites such as Wilkinson Microwave Anisotropy Probe (WMAP) [3] and large scale structure [4]. Astrophysical observations indicate that accelerated expansion of the universe is driven by an exotic energy with large negative pressure which is known as dark energy (DE).

The data indicates that the universe is spatially flat and is dominated by $76 \%$ DE and $24 \%$ other matter (20\% dark matter and $4 \%$ other cosmic matter). Despite all lines of observational evidence, the nature of DE is still a challenging problem in theoretical physics. Several models have been proposed such as, quintessence [5], phantom field [6], tachyon field [7], quintom [8] and the interacting DE models, Chaplygin gas [9], holographic models [10] and braneworld models [11], etc. However, none of these models can be regarded as being entirely convincing so far.

In black hole physics, the temperature and entropy are proportional to the surface gravity at the horizon and the area of the horizon [12, 13], respectively. Also, the temperature, entropy and mass of the black hole satisfy the first law of thermodynamics [14]. This leads to the relationship between the black hole thermodynamics and the Einstein field equations. Jacobson [15] derived the field equations from the first law of thermodynamics for all local Rindler causal horizons. Padmanavan [16] formulated the first law of thermodynamics on the horizon using the field equations for a general static spherically symmetric spacetime. Later, this relationship was developed in the cosmological context by taking universe as a thermodynamical system bounded by the apparent horizon. Cai and Kim [17] showed the equivalence of the first law of thermodynamics to the Friedmann equations with the generalized second law of thermodynamics (GSLT) at the horizon.

Cosmological models in which DE interacts with dark matter and other cosmic matter are well-known in the literature [18]. The discovery of black hole thermodynamics has led to the thermodynamics of cosmological models. Bekenstein [13] proved that there is a relation between an event horizon and thermodynamics of black hole. The event horizon of black hole is in fact the measure of entropy which is generalized to the cosmological models so that each horizon corresponds to an entropy. The GSLT is generalized in such a way that the sum of the time derivative of each entropy must be increasing. People studied the thermodynamics of different models in which different cosmic constituents interact.

Wang et al. [19] found that both the first and the second law of thermodynamics break down at the event horizon. The first law of thermodynamics is restricted to nearby states of local thermodynamic equilibrium while the 
event horizon reflects global features of the spacetime. Also, due to the existence of the cosmological event horizon, the universe should be non-static in nature and, as a result, the usual definition of the thermodynamical quantities on the event horizon may not be as simple as in the static spacetime. Finally, they have argued that as the event horizon is larger than the apparent horizon so the universe bounded by the event horizon is not a Bekenstein system.

There is a large body of literature [20]- 32 ] dealing with the thermodynamics of the universe bounded by the apparent horizon and the validity of GSLT. Mazumder et al. [33], [34] have explored the validity of GSLT using the first law of thermodynamics and found some conditions on its validity. Debnath [35] investigated the validity of GSLT in the scenario of Friedmann Robertson Walker (FRW) model in which DE interacts with dark matter and radiation. Mubasher et al. [36] proved the validity of GSLT for all time, independent of geometry and equation of state (EoS) parameter. In a recent paper [37], the validity of GSLT is explored in the Kaluza-Klein cosmology with modified holographic DE.

Jacobs [38] studied the spatially homogeneous and anisotropic Bianchi type $I$ (BI) cosmological model with expansion and shear but without rotation. He discussed anisotropy in the temperature of CMB and cosmic expansion both with and without magnetic field. Akarsu and Kilinc [39] investigated anisotropic BI models in the presence of perfect fluid and minimally interacting DE with anisotropic EoS parameter. They found that anisotropy of the DE did not always promote the anisotropy in the expansion.

In this paper, we take the BI model in which anisotropic DE interacts with isotropic dark matter as well as anisotropic radiation and investigate the validity of GSLT. The plan of this paper is the following: in next section, we take the BI model as the representation of the universe and evaluate shear parameters $(R, S)$, skew parameters $(\delta, \gamma, \zeta, \xi)$. Also, we formulate the corresponding field equations, the generalized Friedmann equation and equations of continuity in the effective field theory. Section $\mathbf{3}$ presents the analysis of the validity of GSLT. The last section concludes the paper results. 


\section{Anisotropic DE Interacting with Two Flu- ids}

The metric representation of the BI universe is given as

$$
d s^{2}=-d t^{2}+A^{2}(t) d x^{2}+B^{2}(t) d y^{2}+C^{2}(t) d z^{2},
$$

where $A, B, C$ are scale factors along the $x, y, z$ axes respectively. When $A=B=C$, the BI model reduces to the flat FRW model. Thus BI is the generalization of the flat FRW model. The energy-momentum tensor is defined as

$$
T_{\nu}^{\mu}=T_{(m) \nu}^{\mu}+T_{(\Lambda) \nu}^{\mu}+T_{(\chi) \nu}^{\mu},
$$

where the subscripts $m, \Lambda, \chi$ denote dark matter, anisotropic DE and anisotropic radiation, respectively, and corresponding energy momentum tensors are given as follows:

$$
\begin{aligned}
T_{(m) \nu}^{\mu} & =\left(-\rho_{m}, P_{m}, P_{m}, P_{m}\right), \\
T_{(\Lambda) \nu}^{\mu} & =\left(-\rho_{\Lambda}, P_{(\Lambda) x}, P_{(\Lambda) y}, P_{(\Lambda) z}\right), \\
T_{(\chi) \nu}^{\mu} & =\left(-\rho_{\chi}, P_{(\chi) x}, P_{(\chi) y}, P_{(\chi) z}\right) .
\end{aligned}
$$

The corresponding EoSs for these components are

$$
\begin{aligned}
& P_{(\Lambda) x}=\rho_{\Lambda} \omega_{(\Lambda) x}, \quad P_{(\Lambda) y}=\rho_{\Lambda} \omega_{(\Lambda) y}, \quad P_{(\Lambda) z}=\rho_{\Lambda} \omega_{(\Lambda) z} \\
& P_{(m) x}=\rho_{m} \omega_{(m) x}, \quad P_{(m) y}=\rho_{m} \omega_{(m) y}, \quad P_{(m) z}=\rho_{m} \omega_{(m) z} \\
& P_{(\chi) x}=\rho_{\chi} \omega_{(\chi) x}, \quad P_{(\chi) y}=\rho_{\chi} \omega_{(\chi) y}, \quad P_{(\chi) z}=\rho_{\chi} \omega_{(\chi) z} .
\end{aligned}
$$

To parameterize the anisotropy of pressure, we define skewness parameters. These parameters actually measure the deviation in pressure along the $y$ and $z$-axis from the $x$-axis. The skewness parameters for anisotropic DE are defined as [40]

$$
3 \delta=\frac{P_{(\Lambda) x}-P_{(\Lambda) y}}{\rho_{\Lambda}}, \quad 3 \gamma=\frac{P_{(\Lambda) z}-P_{(\Lambda) x}}{\rho_{\Lambda}} .
$$

Using Eqs.(2.6) and (2.9), we can write Eq.(2.4) as follows:

$$
T_{(\Lambda) \nu}^{\mu}=\left[-\rho_{\Lambda}, \omega_{\Lambda} \rho_{\Lambda},\left(\omega_{\Lambda}+3 \delta\right) \rho_{\Lambda},\left(\omega_{\Lambda}+3 \gamma\right) \rho_{\Lambda}\right]
$$


The skewness parameters for anisotropic radiation are

$$
3 \zeta=\frac{P_{(\chi) x}-P_{(\chi) y}}{\rho_{\chi}}, \quad 3 \xi=\frac{P_{(\chi) z}-P_{(\chi) x}}{\rho_{\chi}} .
$$

With the help of Eqs.(2.8) and (2.11), Eq.(2.5) turns out to be

$$
T_{(\chi) \nu}^{\mu}=\left[-\rho_{\chi}, \omega_{\chi} \rho_{\chi},\left(\omega_{\chi}+3 \zeta\right) \rho_{\chi},\left(\omega_{\chi}+3 \xi\right) \rho_{\chi}\right] .
$$

Making use of Eqs.(2.10) and (2.12) in Eq.(2.2), we have

$$
\begin{aligned}
T_{\nu}^{\mu} & =\left[-\rho_{m}, \omega_{m} \rho_{m}, \omega_{m} \rho_{m}, \omega_{m} \rho_{m}\right]+\left[-\rho_{\Lambda}, \omega_{\Lambda} \rho_{\Lambda},\left(\omega_{\Lambda}+3 \delta\right) \rho_{\Lambda},\left(\omega_{\Lambda}+3 \gamma\right) \rho_{\Lambda}\right] \\
& +\left[-\rho_{\chi}, \omega_{\chi} \rho_{\chi},\left(\omega_{\chi}+3 \zeta\right) \rho_{\chi},\left(\omega_{\chi}+3 \xi\right) \rho_{\chi}\right] .
\end{aligned}
$$

The corresponding field equations take the following form:

$$
\begin{aligned}
\frac{\dot{A} \dot{B}}{A B}+\frac{\dot{B} \dot{C}}{B C}+\frac{\dot{C} \dot{A}}{C A} & =\left[\rho_{m}+\rho_{\Lambda}+\rho_{\chi}\right], \\
\frac{\ddot{B}}{B}+\frac{\ddot{C}}{C}+\frac{\dot{B} \dot{C}}{B C} & =-\left[\omega_{m} \rho_{m}+\omega_{\Lambda} \rho_{\Lambda}+\omega_{\chi} \rho_{\chi}\right], \\
\frac{\ddot{A}}{A}+\frac{\ddot{C}}{C}+\frac{\dot{A} \dot{C}}{A C} & =-\left[\omega_{m} \rho_{m}+\left(\omega_{\Lambda}+3 \delta\right) \rho_{\Lambda}+\left(\omega_{\chi}+3 \zeta\right) \rho_{\chi}\right], \\
\frac{\ddot{A}}{A}+\frac{\ddot{B}}{B}+\frac{\dot{A} \dot{B}}{A B} & =-\left[\omega_{m} \rho_{m}+\left(\omega_{\Lambda}+3 \gamma\right) \rho_{\Lambda}+\left(\omega_{\chi}+3 \xi\right) \rho_{\chi}\right] .
\end{aligned}
$$

The continuity equations, $T_{; \nu}^{\mu \nu}=0$, are

$$
\begin{array}{r}
\dot{\rho_{\Lambda}}+H \rho_{\Lambda}\left[3\left(1+\omega_{\Lambda}\right)+\delta(3-2 R+S)+\gamma(3-2 S+R)\right]=-Q^{\prime}, \\
\dot{\rho_{m}}+3 H \rho_{m}\left(1+\omega_{m}\right)=Q .
\end{array}
$$

Here $Q$ and $Q^{\prime}$ are introduced because two principal components of the universe are mutually interacting, which lead to some loss in other cosmic component. We take this component as radiation for which the continuity equation becomes

$$
\dot{\rho}_{\chi}+H \rho_{\chi}\left[3\left(1+\omega_{\chi}\right)+\zeta(3-2 R+S)+\xi(3-2 S+R)\right]=Q^{\prime}-Q .
$$

Here, we define the mean expansion rate as an average Hubble rate $H$ by

$$
H=\frac{1}{3}\left(\frac{\dot{A}}{A}+\frac{\dot{B}}{B}+\frac{\dot{C}}{C}\right) .
$$


The difference of expansion rates as the Hubble normalized shear parameters $R$ and $S$ are given as [41]

$$
R=\frac{1}{H}\left(\frac{\dot{A}}{A}-\frac{\dot{B}}{B}\right), \quad S=\frac{1}{H}\left(\frac{\dot{A}}{A}-\frac{\dot{C}}{C}\right) .
$$

Shear parameters are also deviation measuring parameters which parameterize anisotropy in scale factors by taking expansion along $x$-axis as standard. Here, all expansion rates are positive and $R>-3, S<3$. This is also true either for $R=0$ or $S=0$. The generalized Friedmann equation takes the form [40]

$$
H^{2}=\frac{\rho_{m}+\rho_{\Lambda}+\rho_{\chi}}{3\left(1-\frac{1}{9}\left(R^{2}+S^{2}-R S\right)\right)} .
$$

We take [42] $Q^{\prime}=\Gamma_{\Lambda} \rho_{\Lambda}, Q=\Gamma_{m} \rho_{\Lambda}$. The ratios for energy densities as

$$
r_{1}=\frac{\rho_{m}}{\rho_{\Lambda}}, \quad r_{2}=\frac{\rho_{\chi}}{\rho_{\Lambda}}
$$

The corresponding EoS parameters in the effective field theory will become

$$
\begin{aligned}
\omega_{\Lambda}^{e f f} & =\omega_{\Lambda}+\frac{\Gamma_{\Lambda}}{3 H}+\frac{\delta(3-2 R+S)+\gamma(3-2 S+R)}{3}, \\
\omega_{m}^{e f f} & =\omega_{m}-\frac{\Gamma_{m}}{3 H r_{1}}, \\
\omega_{\chi}^{e f f} & =\omega_{\chi}+\frac{\Gamma_{m}-\Gamma_{\Lambda}}{3 H r_{2}}+\frac{\zeta(3-2 R+S)+\xi(3-2 S+R)}{3} .
\end{aligned}
$$

Consequently, the continuity equations (2.18)-(2.20) turn out to be

$$
\begin{aligned}
\dot{\rho_{\Lambda}}+3 H \rho_{\Lambda}\left(1+\omega_{\Lambda}^{\text {eff }}\right) & =0 \\
\dot{\rho}_{m}+3 H \rho_{m}\left(1+\omega_{m}^{\text {eff }}\right) & =0 \\
\dot{\rho_{\chi}}+3 H \rho_{\chi}\left(1+\omega_{\chi}^{\text {eff }}\right) & =0 .
\end{aligned}
$$

\section{Generalized Second Law Of Thermodynam- ics}

Now we investigate the validity of the GSLT in BI universe bounded by apparent horizon with size $L$ which coincides with Hubble horizon in the 
case of flat geometry, i.e., $L=\frac{1}{H}$. The first law of thermodynamics gives

$$
T d S=P d V+d E, \quad d S=\frac{P d V+d E}{T},
$$

where $T, S, E$ and $P$ are the temperature, entropy, internal energy and pressure of the system respectively. The corresponding entropies will become

$$
d S_{\Lambda}=\frac{P_{\Lambda} d V+d E_{\Lambda}}{T}, \quad d S_{m}=\frac{P_{m} d V+d E_{m}}{T}, \quad d S_{\chi}=\frac{P_{\chi} d V+d E_{\chi}}{T},
$$

where $P_{\Lambda}, P_{\chi}, P_{m}, E_{\Lambda}, E_{\chi}$ and $E_{m}$ are the pressures and internal energies of anisotropic DE, anisotropic radiation and dark matter, respectively. We assume that the system is in equilibrium, which implies that all the components of the system have the same temperature given by [13]

$$
T=\frac{K}{2 \pi},
$$

where $K$ is the surface gravity of the black hole. At equilibrium, the horizon and other components of the system have same temperature. Thermodynamical quantities are related to the cosmological quantities by the following relations:

$$
\begin{aligned}
& P_{\Lambda}=\omega_{\Lambda}^{\text {eff }} \rho_{\Lambda}, \quad P_{\chi}=\omega_{\chi}^{e f f} \rho_{\chi}, \quad P_{m}=\omega_{m}^{\text {eff }} \rho_{m}, \\
& E_{\Lambda}=A B C \rho_{\Lambda}, \quad E_{\chi}=A B C \rho_{\chi}, \quad E_{m}=A B C \rho_{m},
\end{aligned}
$$

where $V=A B C$ is the volume of the system containing all the matter.

The entropy of the horizon is $S_{h}=\frac{k A^{*}}{4}$, where $A^{*}$ is the surface area of the black hole and $k$ is the Boltzmann's constant. Here $A^{*}=4 \pi L^{2}$ so that we have $S_{h}=k \pi L^{2}$, which leads to

$$
\dot{S}_{h}=2 k \pi L \dot{L} .
$$

Also, the time derivative of Eq.(3.30) yields

$$
\dot{S}_{\Lambda}=\frac{P_{\Lambda} \dot{V}+\dot{E}_{\Lambda}}{T}, \quad \dot{S}_{\chi}=\frac{P_{\chi} \dot{V}+\dot{E}_{\chi}}{T}, \quad \dot{S_{m}}=\frac{P_{m} \dot{V}+\dot{E}_{m}}{T} .
$$

Using Eqs.(2.27)-(2.29), (3.34) and (3.35), it follows that

$$
\dot{S}_{\text {total }}=2 k \pi L \dot{L},
$$


where $\dot{S}_{\text {total }}$ is the sum of the matter entropy and horizon entropy. Now making use of Eqs.(2.27)-(2.29) and (2.23), we obtain

$$
\dot{L}=-\frac{\dot{H}}{H^{2}}=\frac{L^{3} H \alpha}{2 \beta}-\frac{L^{3} H^{2}[\dot{R}(2 R-S)+\dot{S}(2 S-R)]}{18 \beta},
$$

where

$$
\begin{aligned}
& \alpha=\left(1+\omega_{\Lambda}\right) \rho_{\Lambda}+\left(1+\omega_{m}\right) \rho_{m}+\left(1+\omega_{\chi}\right) \rho_{\chi} \\
& +\frac{1}{3}\left[\left(\rho_{\Lambda} \delta+\rho_{\chi} \zeta\right)(3-2 R+S)+\left(\rho_{\Lambda} \gamma+\rho_{\chi} \xi\right)(3-2 S+R)\right]
\end{aligned}
$$

and

$$
\beta=1-\frac{R^{2}+S^{2}-R S}{9} \geq 0 .
$$

Inserting this value of $\dot{L}$ in Eq.(3.36), it follows that

$$
\dot{S}_{\text {total }}=\frac{k \pi H L^{4} \alpha}{\beta}-\frac{k \pi H^{2} L^{4}[\dot{R}(2 R-S)+\dot{S}(2 S-R)]}{9 \beta} .
$$

This implies that if $\dot{S}_{\text {total }} \geqslant 0$, then GSLT holds. Thus the validity of GSLT depends upon the skewness, shear and state parameters. From Eq.(3.40), $\dot{S}_{\text {total }} \geqslant 0$ implies that

$$
\frac{k \pi H L^{4} \alpha}{\beta}-\frac{k \pi H^{2} L^{4}[\dot{R}(2 R-S)+\dot{S}(2 S-R)]}{9 \beta} \geq 0 .
$$

After substituting the values of $\alpha$ and $\beta$ in the above equation, it follows that

$$
\begin{aligned}
& 9(\rho+p) \geq[\dot{R}(2 R-S)+\dot{S}(2 S-R)]-3\left[\left(\rho_{\Lambda} \delta+\rho_{\chi} \zeta\right)(3-2 R+S)\right. \\
& \left.+\left(\rho_{\Lambda} \gamma+\rho_{\chi} \xi\right)(3-2 S+R)\right] .
\end{aligned}
$$

Now, we consider the following three interesting cases involving the condition on different parameters:

I. $\beta=0$, II. $\beta>0$, III. $R=S=\delta=\gamma=\xi=\zeta=0$.

\section{Case I}

Here $H$ and $\dot{S}_{\text {total }}$ tend to infinity for $\beta=0$ from Eqs.(2.23) and (3.40) respectively. It may happen for very large time $(t \rightarrow \infty)$, i.e., when the 
expansion rate is very high. In this case, all the useable energy in the universe will be converted into another form of energy which is not useable. This stage is also known as the heat death of the system. The heat death of the universe is said to be a suggested fate of the universe. At this time, all the thermodynamic free energy will be diminished from the universe and motion or life cannot sustain any more. In the language of physics, the entropy in the universe will reach to its maximum value.

\section{Case II}

In this case, by takeing $\beta>0$ in Eq.(3.40), we observe that the validity of this law depends on the skewness, shear and EoS parameters. Further, if we remove anisotropy in the expansion, its validity depends on skewness as well as equation of state parameters, and if we remove anisotropy in fluid, then it depends on shear and equation of state parameters. Hence, GSLT is conditionally valid.

\section{Case III}

When we take $R=S=\delta=\gamma=\xi=\zeta=0$, BI reduces to the flat FRW model. Using these values in Eq.(3.41), we have $(\rho+p) \geq 0$, which is the null energy condition in the FRW universe. Consequently, Eq. (3.40) leads to $\dot{S}_{\text {total }}=k \pi(\rho+p)$ and hence $\dot{S}_{\text {total }} \geq 0$ for $(\rho+p) \geq 0$. Thus we can say that GSLT holds for all time in this case, if the null energy condition for the considered matter is satisfied in the FRW universe. This case implies that the validity of GSLT depends on the null energy condition which is not discussed in [36]. Therefore using results produced in this case, we can say that the validity of GSLT depends on null energy condition and background geometry.

\section{Conclusion}

This paper is devoted to study the validity of GSLT with the BI universe model. We assume that anisotropic DE is interacting with dark matter and anisotropic radiation. Using this scenario, we have formulated the conditions under which GSLT is valid. We have discussed three cases for its validity. For a particular value of $\beta=0$, the first case provides the general validity 
of GSLT. In this case, we see that at infinite expansion the heat death of universe will take place. At this stage all types of motion and life will be finished. The second case $\beta>0$ gives conditional validity of this law. In the third case, BI reduces to the flat FRW metric and the general validity of GSLT depends upon the condition $(\rho+p) \geq 0$, which is the null energy condition. We would like to mention here that the validity of this law for the FRW universe with the same scenario has been proved [36] independent of background geometry, EoS parameter and interacting scenario. This analysis indicates that the validity of GLST for FRW depends on geometry as well as null energy condition. We conclude that GSLT is conditionally valid in the BI type universe with anisotropic perfect fluid.

\section{References}

[1] Perlmutter, S. et al.: Science 284(1999)1481; Perlmutter, S. et al.: Nature 391(1998)51.

[2] Riess, A.G. et al.: Astron. J. 116(1998)1009; Perlmutter, S. et al.: Astrophys. J. 517(1999)565.

[3] Bennett, C.L. et al.: Astrophys. J. Suppl. 148(2003)1; Spergel, D.N. et al.: Astrophys. J. Suppl. 148(2003)175.

[4] Verde, L. et al.: Mon. Not. R. Astron. Soc. 335(2002)432; Hawkins, E. et al.: Mon. Not. Roy. Astr. Soc. 346(2003)78; Abazajian, et al.: Phys. Rev. D69(2004)103501.

[5] Sahni, V. and Starobinsky, A.A.: Int. J. Mod. Phys. D9(2000)373; Sahni, V.: Lect. Notes Phys. 653(2004)141; Padmanabhan, T.: Gen. Relativ. Gravit. 40(2008)529.

[6] Caldwell, R.R.: Phys. Lett. B545(2002)23; Nojiri, S. and Odintsov, S.D.: Phys. Lett. B562(2003)147; ibid. B565(2003)1.

[7] Sen, A.: JHEP 04(2002)048; Padmanabhan, T.: Phys. Rev. D66(2002)021301; Padmanabhan, T. and Choudhury, T.R.: Phys. Rev. D66(2002)081301.

[8] Feng, B.,Wamg, X.L. and Zhang, X.M.: Phys. Lett. B607(2005)35; Guo, Z.K. et al.: Phys. Lett. B608(2005)177. 
[9] Kamenshchik, A., Moschella, U. and Pasquier, V.: Phys. Lett. B511(2001)265; Bento, M.C., Bertolami, O. and Sen, A.A.: Phys. Rev. D66(2002)043507.

[10] Wang, B., Gong, Y.G. and Abdalla, E.: Phys. Lett. B624(2005)141; Setare, M.R.: Phys. Lett. B642(2006)1; Hu, B. and Ling, Y.: Phys. Rev. D73(2006)123510; Kim, H., Lee, H.W. and Myung, Y.S.: Phys. Lett. B632(2006)605; Setare, M.R.: JCAP 0701(2007)023.

[11] Li, M.: Phys. Lett. B603(2004)1; Deffayet, C., Dvali, G.R. and Gabadadze, G.: Phys. Rev. D65(2002)044023.

[12] Hawking, S.W.: Commun. Math. Phys. 43(1975)199.

[13] Bekenstein, J.D.: Phys. Rev. D7(1973)2333.

[14] Bardeen, J.M., Carter, B. and Hawking, S.W.: Commun. Math. Phys. 31(1973)161.

[15] Jacobson, T.: Phys. Rev. Lett. 75(1995)1260.

[16] Padmanavan, T.: Class. Quantum Grav. 19(2002)5387.

[17] Cai, R.G. and Kim, S.P.: JHEP 02(2005)050.

[18] Amendola, L.: Phys. Rev. D62(2000)043511; Chimento, L.P., Jakubi, A.S. and Pavon, D.: Phys. Rev. D62(2000)062508; Zimdahl, W., Pavon, D. and Chimento, L.P.: Phys. Lett. B521(2001)133; Chimento, L.P, Jakubi A.S., Pavon D. and W. Zimdahl: Phys. Rev. D67(2003)083513; Farrar, G.R. and Peebles, P.J.E.: Astrophys. J.604(2004)1; Del Campo, S., Herrera, R. and Pavon, D.: Phys. Rev. D70(2004)043540; Del Campo, S., Herrera, R. and Pavon, D.: Phys. Rev.D71(2005)123529.

[19] Wang, B., Gong, Y. and Abdalla, E.: Phys. Rev. D74(2006)083520.

[20] Wang, B., Gong, Y. and Abdalla, E.: Phys. Lett. B652(2007)86.

[21] Saridakis, E.N. and Setare, M.R.: Phys. Lett. B670(2008)01.

[22] Saridakis, E.N.: Phys. Lett. B661(2008)335.

[23] Charmouis, C. and Dufaux, J.F.: Class. Quantum Grav. 19(2002)4671. 
[24] Kim, J.E., Kyae, B. and Lee, H.M.: Nucl. Phys. B582(2000)296.

[25] Gravanis, E. and Willison, S.: Phys. Lett. B562(2003)118.

[26] Cai, R.G., Zhang, H.S. and Wang, A.: Commun. Theor. Phys. 44(2005)948.

[27] Bousso, R.S.: Phys. Rev. D71(2005)064024.

[28] Bandyopadhyay, T. and Debnath, U.: arXiv:1105.5301.

[29] Mazumder, N. and Chakraborty, S.: Eur. Phys. C70(2010)329.

[30] Farajollahi, F., Salehi, A. and Tayebi, F.: Can. J. Phys. 89(2011)915.

[31] Das, A., Chattopadhyay, S. and Debnath, U.: arXiv:1104.2378.

[32] Debnath, U. and Mubasher, J.: Astrophys. Space Sci. 335(2011)545.

[33] Mazumder, N. and Chakraborty, S.: Class. Quantum Gravity 26(2009)195016.

[34] Mazumder, N. and Chakraborty, S.: Gen. Relativ. Gravit. 42(2010)813.

[35] Debnath, U.: arXiv:1006.2217.

[36] Mubasher, J., Saridakis, E.N. and Setare, R.M.: Phys. Rev. D81(2010)023007.

[37] Sharif, M. and Khanum, F.: Gen. Relativ. Gravit. 43(2011)2885.

[38] Jacobs, K.C.: Astrophys. J. 153(1968)661; ibid. 155(1969)379.

[39] Akarsu, O. and Kilinc, C.B.: Gen. Relativ. Gravit. 42(2010)763.

[40] Koivisto, T. and Mota, D.F.: JCAP 0806(2008)018.

[41] Barrow, J.D.: Phys. Rev. D55(1997)7451.

[42] Setare, M.R.: JCAP 01(2007)023; Setare, M.R. and Shafei, S.: JCAP 09(2006)011. 